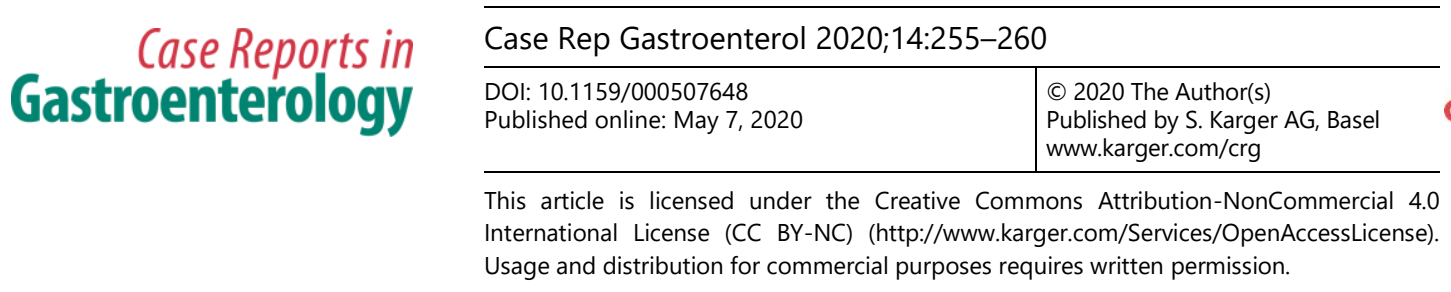

\title{
Fatal Spontaneous Tumor Lysis Syndrome in a Patient with Metastatic Colon Cancer: A Clinical Case of Rare Oncological Emergency
}

\author{
Inna Shaforostova ${ }^{a}$ Robert Fiedler ${ }^{b}$ Martina Zander ${ }^{c}$ \\ Johannes Pflumm ${ }^{d}$ Wolfgang Josef März ${ }^{d}$ \\ aDepartment of Haematology, Haemostasiology, Oncology and Pneumology, Albert-

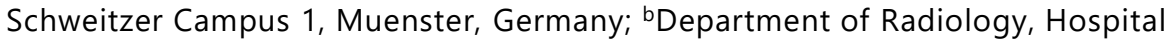 \\ Harzklinikum Dorothea Christiane Erxleben $\mathrm{GmbH}$, Wernigerode, Germany; 'Department \\ of Pathology, Hospital Harzklinikum Dorothea Christiane Erxleben $\mathrm{GmbH}$, \\ Quedlinburg, Germany; dDepartment of Internal Medicine, Hospital Harzklinikum \\ Dorothea Christiane Erxleben $\mathrm{GmbH}$, Wernigerode, Germany
}

\section{Keywords}

Tumor lysis syndrome · Colon cancer · Spontaneous tumor lysis syndrome

\begin{abstract}
Tumor lysis syndrome (TLS) is a potentially life-threatening complication of chemotherapy. It usually occurs in rapidly proliferating hematological malignancies. TLS is deemed spontaneous (STLS) when it occurs prior to any cytotoxic or definite treatment. STLS is extremely rare in solid tumors. Here, we report a rare case of fatal STLS in a 47-year-old woman diagnosed with metastatic colon cancer. The patient developed acute renal failure with anuria, electrolyte disturbances, and metabolic acidosis before initiating chemotherapy. Despite appropriate management of TLS, including renal replacement therapy, she died within a few days from multiorgan failure. Only few other case reports of STLS associated with colon cancer have been reported in the literature.




\section{Case Reports in Gastroenterology}

Case Rep Gastroenterol 2020;14:255-260

DOI: $10.1159 / 000507648$

(c) 2020 The Author(s). Published by S. Karger AG, Basel www.karger.com/crg

Shaforostova et al.: Spontaneous Tumor Lysis Syndrome in Metastatic Colon Cancer

\section{Introduction}

Tumor lysis syndrome (TLS) is an oncologic emergency, mostly occurring in hematological malignancies following the initiation of chemotherapy. TLS is caused by the destruction of tumor cells and subsequent release of intracellular material into the circulation leading to lifethreatening metabolic disturbances (hyperkalemia, hyperuricemia, hyperphosphatemia, hypocalcemia), acute renal failure, and organ dysfunction. Treatment measures include aggressive hydration, use of hypouricemic agents, such as allopurinol and rasburicase, management of electrolyte abnormalities, and, in case of kidney failure, renal replacement therapy. TLS is deemed spontaneous (STLS) when it occurs before any cytotoxic or definite treatment [1]. STLS happens mostly in patients with acute leukemias and aggressive lymphomas. In solid tumors, it occurs very rarely. Herein, we present a case of fatal STLS in a patient diagnosed with metastatic colon cancer.

\section{Case Report}

A 47-year-old Caucasian female was admitted to a community hospital with a 4-week history of abdominal distension, lower extremity swelling, and dyspnea on exertion. Additionally, she had gained $15 \mathrm{~kg}$ over the previous several weeks. Her medical history included an erysipelas of the right leg 5 months before presentation. Her family history was unremarkable. Clinical examination revealed anasarca and distended abdomen with signs of ascites. Laboratory tests were notable for anemia (hemoglobin $6,1 \mathrm{mmol} / \mathrm{L}$ ), an elevated uric acid level (724 $\mu \mathrm{mol} / \mathrm{L}$ ), elevated liver enzymes (total bilirubin 31,3 $\mu \mathrm{mol} / \mathrm{L}$, alkaline phosphatase 5,17 $\mu \mathrm{kat} / \mathrm{L}$, aspartate aminotransferase 3,07 $\mu \mathrm{kat} / \mathrm{L}$ and lactate dehydrogenase activity 15,8 $\mu \mathrm{kat} / \mathrm{L})$, increased white blood cell count $\left(19,1 \times 10^{9} / \mathrm{L}\right)$, and C-reactive protein level $(199,8$ $\mathrm{mg} / \mathrm{L}$ ). Her kidney function was only mildly impaired (creatinine $78 \mu \mathrm{mol} / \mathrm{L}$, eGFR 73 $\mathrm{mL} / \mathrm{min} / 1.73 \mathrm{~m}^{2}$ ). Abdominal ultrasound revealed massive ascites and multiple liver masses. Diagnostic and therapeutic paracentesis was performed. However, ascitic fluid cytology did not reveal tumor cells. A CT scan of the chest, abdomen, and pelvis demonstrated a large pelvic mass, innumerable lesions throughout the liver, peritoneal carcinomatosis with ascites, and multiple bilateral pulmonary nodules (Fig. 1). Her tumor markers were markedly elevated (CEA 3,724 $\mu \mathrm{g} / \mathrm{L}$ and CA125 1,030 U/mL). Based on these findings, the patient was initially suspected of having advanced ovarian cancer. On day 9 , she developed acute renal failure with creatinine at $198 \mu \mathrm{mol} /$ and eGFR $25 \mathrm{~mL} / \mathrm{min} / 1.73 \mathrm{~m}^{2}$ (Fig. 2a).

An ultrasound-guided liver biopsy was obtained on the following day. The preliminary pathology report showed poorly differentiated adenocarcinoma most consistent with a colorectal primary. The Ki-67 proliferation index was high (60\%). On day 12, the patient's condition worsened, she developed metabolic acidosis, hyperkalemia (potassium 5,27 mmol/L), and hypocalcemia (calcium 2,04 mmol/L), hyperphosphatemia (phosphorus 2,76 mmol/L), as well as hyperuricemia $(1,631 \mu \mathrm{mol} / \mathrm{L})$, and extremely high LDH level: $28,15 \mu \mathrm{kat} / \mathrm{L}$ (Fig. 2a). Based on these abnormalities, a presumptive diagnosis of STLS was made. She was transferred to the intensive care unit.

STLS was treated with aggressive intravenous hydration, loop diuretics, febuxostat, phosphate binders, sodium bicarbonate, and rasburicase. Palliative platinum-based chemotherapy was also initiated.

Over the course of the next 2 days, she became anuric with refractory hyperkalemia (potassium 5,90 mmol/L), severe metabolic acidosis, and increasing creatinine level (344 
$\mu \mathrm{mol} / \mathrm{L}$ ). Renal replacement therapy with continuous veno-venous hemofiltration (CVVH) was started on day 15.

Despite aggressive supportive care, the patient's condition continued to deteriorate. She died from multiorgan failure 20 days after admission to the hospital.

The autopsy confirmed a moderately differentiated adenocarcinoma of the caecum with metastases to regional lymph nodes, liver, lungs, both ovaries, and peritoneal carcinomatosis. Furthermore, massive tumor necrosis in the organ and lymph node metastases was revealed, explaining rapidly progressing STLS (Fig. 2b).

\section{Discussion}

TLS is a potentially life-threatening complication of malignant neoplasms after systemic treatment. It was first described in 1929 by Bedrna und Polcak. TLS usually occurs in 12-72 h after initiation of treatment and is characterized by a constellation of certain laboratory abnormalities: hyperkalemia, hyperuricemia, hyperphosphatemia hypocalcemia, and metabolic acidosis. Clinical manifestations include seizures, tetany, cardiac arrhythmias, acute renal failure, and possibly sudden death [2]. The Cairo-Bishop classification defines laboratory (asymptomatic) and clinical (symptomatic) TLS [2].

TLS is observed most frequently in patients with hematologic malignancies such as acute leukemia and non-Hodgkin lymphoma. In different studies, the risk for the development of TLS in this patient group varies from 4 to $42 \%$ [3]. Cytotoxic chemotherapy, antibody/immunotherapy, radiotherapy, and glucocorticoids can trigger TLS [3].

STLS occurs without any triggering factors [4]. It is a very rare complication with an incidence of less than $0.3 \%$ [5]. The most important risk factors for STLS are hematological malignancies, large tumor burden, high tumor cell proliferation rate, pre-existing renal impairment, high lactate dehydrogenase, potassium and phosphate levels, reduced fluid intake, and use of nephrotoxic medication [6].

STLS is mainly observed in aggressive hematological tumors. There are only a few publications on STLS in solid neoplasms. In many of these cases, liver involvement is described as an additional risk factor $[1,7]$. This may be due to a high purine pool in the liver which can be released during necrosis or impaired uric acid metabolism if hepatic function is compromised by a high tumor burden [7]. Overall, TLS has a poor prognosis with mortality of $20-50 \%$ if it is undiagnosed or if diagnosed too late [8].

Current guidelines distinguish between prophylaxis and treatment of TLS [9]. In both cases, close monitoring of electrolytes (potassium, calcium, phosphate), uric acid, LDH, and creatinine is mandatory.

In high-risk patients, prophylactic measures such as intravenous hydration, administration of uricostatic drugs (allopurinol or febuxostat), and/or recombinant urate oxidase (rasburicase) should be started immediately. [10]. Due to the risk of calcium phosphate precipitation and xanthine-obstructive nephropathy, the use of sodium bicarbonate is currently no longer recommended. The management of clinical TLS includes additionally phosphate binders for hyperphosphatemia and renal replacement therapy in case of refractory hyperkalemia and oliguria/anuria. Hypocalcemia requires therapeutic intervention only in symptomatic patients and after prior correction of hyperphosphatemia [9].

In our clinical case, the patient with the diagnosis of metastatic colon cancer developed STLS that could be diagnosed on the basis of the typical laboratory changes (hyperuricemia, hyperphosphatemia, hypocalcemia, and metabolic acidosis) and the acute renal failure. The 
risk factors for STLS in this case were initially LDH elevation, mildly impaired renal function, high tumor burden, extensive liver metastasis, and a very high Ki-67 level.

Our literature review yielded six other published cases of STLS in colon cancer [1,11-15]. The median age at the time of diagnosis was 48,5 years. All patients had metastatic disease with liver involvement. Three patients had additionally multiple lymph node metastases. In four of six cases, LDH was significantly elevated. A high level of uric acid was detected in 5 of 6 patients. Two patients received chemotherapy, in one case with FOLFOX- 6 and bevacizumab, in another case CHOP was started empirically because of concerns for a possible concurrent lymphoma. Each of these cases was treated with aggressive supportive measures. Three patients received rasburicase, and in three cases renal replacement therapy was initiated. The mortality rate was $67 \%$ among the six cases. All four fatal cases, as with our patient, were associated with acute clinical presentation and irreversible multiorgan failure over a few days.

In conclusion, STLS is a rare oncological emergency, which should be considered even in patients with nonhematologic malignancies with risk factors such as high tumor burden with high LDH level and Ki-67 expression, electrolyte imbalances, and acute renal failure. These patients have a very rapid clinical course similar to that of aggressive non-Hodgkin lymphomas, and should be managed accordingly. Only the prompt recognition and aggressive management of TLS in these situations can improve the patient's prognosis and reduce early mortality.

\section{Acknowledgement}

We would like to thank the family of the patient for allowing us to share the details of the clinical history. We would also like to thank Prof. Annalen Bleckmann for revising this article.

\section{Statement of Ethics}

The authors confirm that the parents of the patient have given their written informed consent to publish the case report as well as pictures and tables.

\section{Disclosure Statement}

The authors have conflicts of interest to declare. The authors declare that they have no competing interests.

\section{Funding Sources}

The authors declare that this work was not supported by any grants or funding. 
Shaforostova et al.: Spontaneous Tumor Lysis Syndrome in Metastatic Colon Cancer

\section{Author Contributions}

I. Shaforostova, J. Pflumm: data acquisition, drafting of the manuscript. M. Zander: contribution with pathological slides and relative content. R. Fiedler: contribution with CT slides and relative content.

\section{References}

1 Sommerhalder D, Takalkar AM, Shackelford R, Peddi P. Spontaneous tumor lysis syndrome in colon cancer: a case report and literature review. Clin Case Rep. 2017 Nov;5(12):2121-6.

2 Cairo MS, Bishop M. Tumour lysis syndrome: new therapeutic strategies and classification. Br J Haematol. 2004 Oct;127(1):3-11.

3 Coiffier B, Altman A, Pui CH, Younes A, Cairo MS. Guidelines for the management of pediatric and adult tumor lysis syndrome: an evidence-based review. J Clin Oncol. 2008 Jun;26(16):2767-78.

4 Jasek AM, Day HJ. Acute spontaneous tumor lysis syndrome. Am J Hematol. 1994 Oct;47(2):129-31.

5 Mott FE, Esana A, Chakmakjian C, Herrington JD. Tumor lysis syndrome in solid tumors. Support Cancer Ther. 2005 Apr;2(3):188-191. https://doi.org/10.3816/SCT.2005.n.012.

6 Mirrakhimov AE, Ali AM, Khan M, Barbaryan A. Tumor lysis syndrome in solid tumors: an up to date review of the literature. Rare Tumors. 2014 Jun;6(2):5389.

7 Gemici C. Tumour lysis syndrome in solid tumours. Clin Oncol (R Coll Radiol). 2006 Dec;18(10):773-80.

8 Coiffier B. Acute tumor lysis syndrome - a rare complication in the treatment of solid tumors. Onkologie. 2010;33(10):498-9.

9 Jones GL, Will A, Jackson GH, Webb NJ, Rule S; British Committee for Standards in Haematology. Guidelines for the management of tumour lysis syndrome in adults and children with haematological malignancies on behalf of the British Committee for Standards in Haematology. Br J Haematol. 2015 Jun;169(5):661-71.

10 Weeks AC, Kimple ME. Spontaneous Tumor Lysis Syndrome: A Case Report and Critical Evaluation of Current Diagnostic Criteria and Optimal Treatment Regimens. J Investig Med High Impact Case Rep. 2015 Aug;3(3):2324709615603199.

11 Berringer R. Spontaneous tumor lysis syndrome in a patient with newly diagnosed metastatic colonic adenocarcinoma. CJEM. 2018 Oct;20 S2:S41-3.

12 Frestad D, Perner A, Pedersen UG. Acute onset and rapid progression of multiple organ failure in a young adult with undiagnosed disseminated colonic adenocarcinoma. BMJ Case Rep. 2014 Sep:bcr2014205002.

13 Kearney MR, Chen EY, Stenzel P, Corless C, Deloughery T, Zivney M. at al. Colorectal Cancer-Associated Spontaneous Tumor Lysis Syndrome: a Case Report and Review of the Current Literature. J Gastrointest Cancer. 2018 Apr;50:668-73. https://doi.org/10.1007/s12029-018-0102-7.

14 Shah SM, Rosenthal MH, Griffin GK, Jacobsen ED, McCleary NJ. An aggressive presentation of colorectal cancer with an atypical lymphoproliferative pattern of metastatic disease: a case report and review of the literature. Clin Colorectal Cancer. 2014 Sep;13(3):e5-11.

15 Vaisban E, Braester A, Mosenzon O, Kolin M, Horn Y. Spontaneous tumor lysis syndrome in solid tumors: really a rare condition? Am J Med Sci. 2003 Jan;325(1):38-40. 
Shaforostova et al.: Spontaneous Tumor Lysis Syndrome in Metastatic Colon Cancer

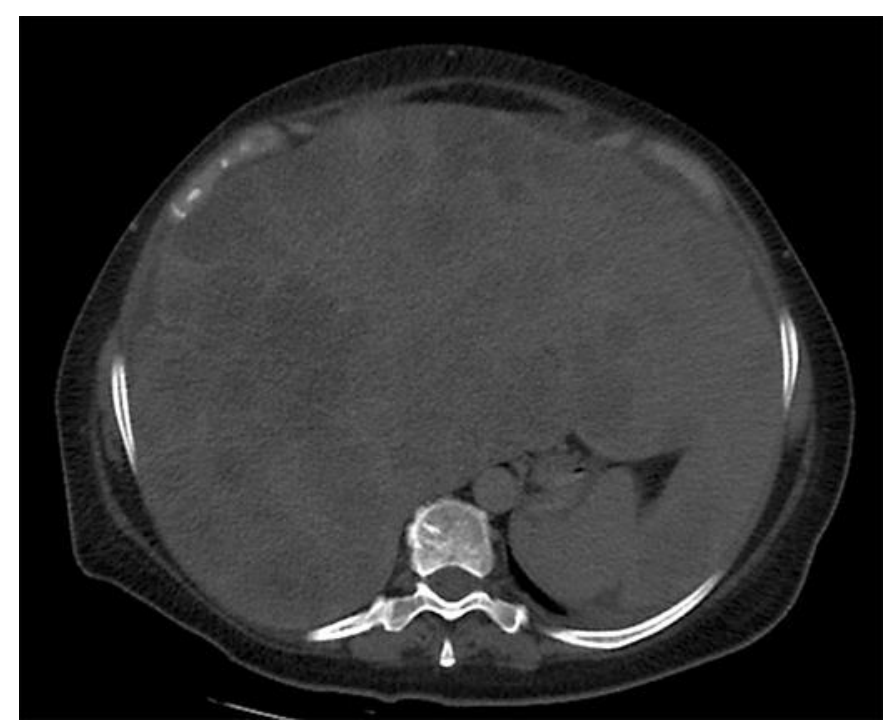

Fig. 1. Abdominal non-contrast CT scan demonstrating multiple liver metastases.

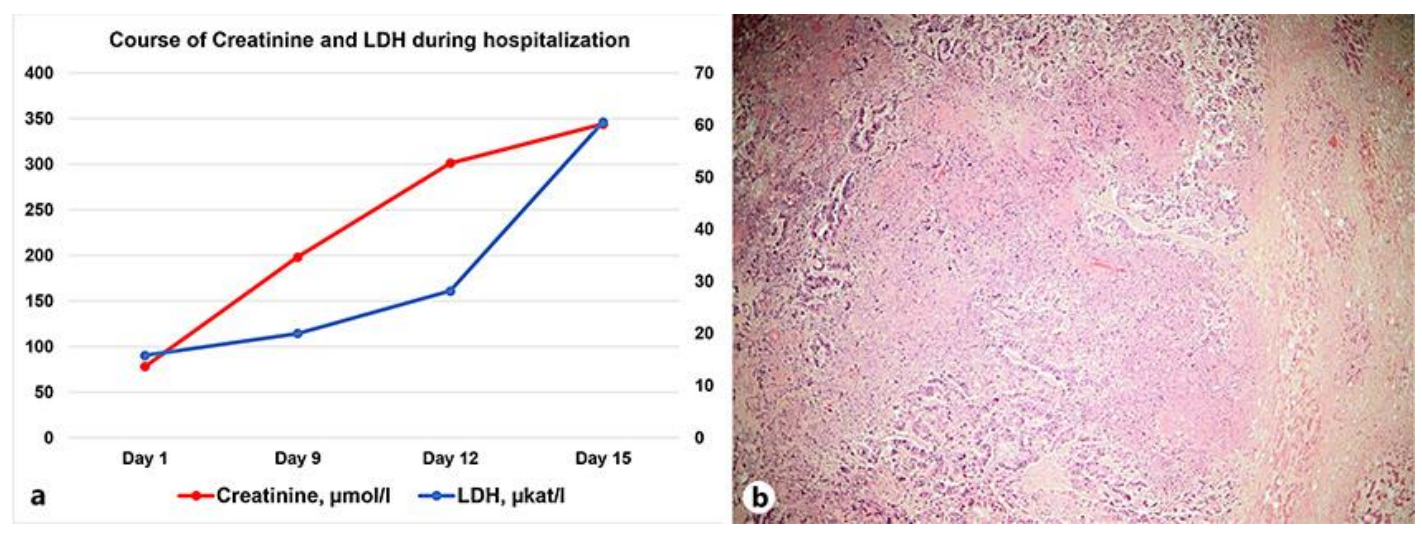

Fig. 2. a Course of creatinine and LDH during hospitalization. b Liver autopsy: moderately differentiated colonic adenocarcinoma with extensive areas of necrosis $(H \& E$, magnification $\times 20)$. 